\title{
Exenatide mitigates inflammation and hypoxia along with improved angiogenesis in obese fat tissue
}

\author{
Yingxin Xian1,2,*, Zonglan Chen1,2,*, Hongrong Deng1,2, Mengyin Cai1,2, Hua Liang1,2, Wen Xu1,2, \\ Jianping Weng1,2 and Fen $\mathrm{Xu}^{1,2}$ \\ 'Department of Endocrinology and Metabolism, The Third Affiliated Hospital of Sun Yat-sen University, Guangzhou, China \\ ${ }^{2}$ Guangdong Provincial Key Laboratory of Diabetology, Guangzhou, China
}

Correspondence should be addressed to J Weng or F Xu: wjianp@mail.sysu.edu.cn or xufen3@mail.sysu.edu.cn

*(Y Xian and Z Chen contributed equally to this work)

\begin{abstract}
Obesity-associated chronic inflammation in adipose tissue is partly attributed to hypoxia with insufficient microcirculation. Previous studies have shown that exenatide, a glucagon-like peptide 1 (GLP-1) receptor agonist, plays an anti-inflammatory role. Here, we investigate its effects on inflammation, hypoxia and microcirculation in white adipose tissue of diet-induced obese (DIO) mice. DIO mice were injected intraperitoneally with exenatide or normal saline for 4 weeks, while mice on chow diet were used as normal controls. The mRNA and protein levels of pro-inflammatory cytokines, hypoxia-induced genes and angiogenic factors were detected. Capillary density was measured by laser confocal microscopy and immunochemistry staining. After 4-week exenatide administration, the dramatically elevated pro-inflammatory cytokines in serum and adipose tissue and macrophage infiltration in adipose tissue of DIO mice were significantly reduced. Exenatide also ameliorated expressions of hypoxia-related genes in obese fat tissue. Protein levels of endothelial markers and pro-angiogenic factors including vascular endothelial growth factor and its receptor 2 were augmented in accordance with increased capillary density by exenatide in DIO mice. Our results indicate that inflammation and hypoxia in adipose tissue can be mitigated by GLP-1 receptor agonist potentially via improved angiogenesis and microcirculation in obesity.
\end{abstract}
Key Words
- GLP-1
- obesity
- inflammation
- hypoxia
- angiogenesis

\section{Introduction}

Obesity is widely considered as a state with low-grade chronic inflammation (de Heredia et al. 2012). Elevated circulating levels of inflammatory cytokines, such as tumour necrosis factor (TNF)-alpha, interleukin (IL)-6, plasminogen activator inhibitor (PAI) and C-reactive protein (CRP), have been reported in adults with obesity; and the elevated levels of IL-1beta and IL- 6 are predictive of type 2 diabetes (T2D) (Donath \& Shoelson 2011). Besides, the discovery that adipose tissue from humans with obesity and obese mice models is infiltrated with increased numbers of macrophages provides a major mechanistic advance into understanding obesityassociated inflammation (Olefsky \& Glass 2010). These adipose tissue macrophages are a major source of 
pro-inflammatory cytokines such as IL-6 and TNF-alpha, which can function in a paracrine and potentially an endocrine fashion to cause decreased insulin sensitivity (Weisberg et al. 2003).

Experimental evidence has proved that increased expressions of inflammatory genes could be caused by hypoxia (Ye et al. 2007), and macrophages were found to predominantly accumulate in hypoxic areas in adipose tissue of obese mice (Rausch et al. 2008). These findings indicate that the trigger of obesity-associated inflammation is hypoxia in expanding adipose tissue. Previous studies showed that the physiological basis of adipose tissue hypoxia might be related to reduction in adipose tissue blood flow and capillary density (Ye 2011). Normally, adipose tissue is highly vascularized with multiple capillaries in contact with each adipocyte (Christiaens \& Lijnen 2010). The increase in body fat should be accompanied by an increase in vascularization to provide adequate oxygen and nutrients (Nishimura et al. 2007). However, it is not always the case. It has been proposed that insufficient neovascularization during the progressive development of obesity may lead to a relative oxygen deficit in adipose tissue (Gealekman et al. 2011). A reduction in blood flow may be a result of such angiogenic failure (Karpe et al. 2002). In addition, blood perfusion is reduced due to impaired vasomotor function, evidenced by the finding that arterioles isolated from visceral omental fat in subjects with obesity exhibit severely impaired endothelium-dependent vasodilation (Farb et al. 2012).

Clinical studies demonstrated that exenatide, a glucagon-like peptide 1 (GLP-1) receptor agonist, improved glycemic control and reduced body weight in patients with T2D (Nauck et al. 2007, Zinman et al. 2007, Shyangdan et al. 2010). Accumulating data from both clinical and animal studies has shown potential anti-inflammatory effect of GLP-1 receptor agonist on the cellular and molecular level (Lee \& Jun 2016). A series of studies conducted in patients with obesity showed that exenatide suppressed oxidative stress and the expression of inflammatory mediators including monocyte chemoattractant proteins (MCP)-1, TNF-alpha and IL-1beta (Wu et al. 2011, Chaudhuri et al. 2012), but the underlying mechanism remained unknown. Recent studies indicated that GLP-1 acutely recruited muscle microvasculature in skeletal muscles, expanded muscle microvascular surface area and improved insulin's metabolic action in the insulin-resistant state (Chai et al. 2012, 2014). Based on the relationship between obesity-related chronic inflammation and hypoxia caused by insufficient angiogenesis in adipose tissue, we investigated the actions of GLP-1 on inflammation, hypoxia and microvasculature in white adipose tissue of diet-induced obese (DIO) mice.

\section{Materials and methods}

\section{Animal study}

Seven-week-old male C57BL/6J mice were purchased from Model Animal Research Center of Nanjing University (Nanjing, China). They were maintained on a 12-h light/ dark cycle with free access to food and water. After oneweek acclimatization, mice were randomly distributed into two initial groups fed with a standard chow diet (NC, 4\% fat wt/wt, Guangdong Medical Laboratory Animal Center) or a high-fat diet (HFD, 34.9\% fat wt/wt, D12492, Research Diets) for 12 weeks. The ones with HFD feeding were then further divided into two groups, one group with exenatide treatment (HFD+Exe group) and another one with normal saline as control (HFD group). Exenatide (Eli Lilly) was injected intraperitoneally $(24 \mathrm{nmol} / \mathrm{kg}$ daily). Mice in the normal control (NC) group were also injected with normal saline every day. After 4 weeks exenatide administration, blood and epididymal fat tissues were collected. Each group consisted of five mice. All protocols and procedures for animal study were approved by the Institutional Animal Care and Use Committee (IACUC) of Sun Yat-sen University.

\section{Tolerance tests and metabolic measurements}

The intraperitoneal (i.p.) glucose tolerance test (i.p.GTT) and insulin tolerance test (i.p.ITT) were carried out after 4-week exenatide intervention. For i.p.GTT, mice were fasted overnight and were given $2 \mathrm{~g}$ glucose $/ \mathrm{kg}$ body weight via i.p. injection. For i.p.ITT, mice were fasted for $6 \mathrm{~h}$ and were i.p. injected with recombinant human insulin Novolin R (Novo Nordisk) $(0.65 \mathrm{U} / \mathrm{kg}$ ). Tail vein blood glucose level was measured at $0,30,60,90,120 \mathrm{~min}$ both in i.p.GTT and i.p.ITT.

\section{Serum analysis}

After $6 \mathrm{~h}$ fasting, C57BL/6 mice were killed and blood samples were collected. Serum samples were separated by centrifugation at $4^{\circ} \mathrm{C}$ and stored at $-80^{\circ} \mathrm{C}$ until measurements were performed. Serum inflammatory adipokines, including IL-6, PAI and TNF-alpha, were measured using a MENDO-75K kit (Millipore). 


\section{Vascular imaging}

Epididymal fat staining was performed as previously reported ( $\mathrm{Xu}$ et al. 2012). Mice were killed and the tip portion of epididymal fat was collected under sterile condition and minced into small pieces $(2-3 \mathrm{~mm})$ using scissors. The fat tissue pieces were washed with $1 \times$ PBS once and then incubated in PBS containing both Griffonia simplicifolia IB4 isolectin Alexa Fluor $488(40 \mu \mathrm{g} / \mathrm{mL}$ in PBS) (I21411, Invitrogen, Thermo Fisher) to stain endothelial cells and BODIPY 558/568 ( $5 \mu \mathrm{M}$ in PBS) (D-3835, Invitrogen, Thermo Fisher) for lipid staining overnight. The samples were washed three times in PBS (5-10 min per time) and fixed with $4 \%$ (wt/vol) formaldehyde for $24 \mathrm{~h}$ for long-term storage. Samples were kept in $1 \times$ PBS during the imaging process.

\section{Confocal microscopy and image analysis}

Vascular images were collected using a Zeiss 510 META confocal microscope with appropriate excitation lasers and band pass filters and equipped with a 20x PlanApochromat objective lens (NA, 0.8). Image stacks were collected with a frame average of two and a step size of $0.76 \mu \mathrm{m}$. At least five randomly selected data stacks were collected for each sample. To quantify the capillary density, the fluorescent signal was collected with confocal microscope at multiple z-planes in the tissue and then to generate $3 \mathrm{D}$ reconstructions. Generation of 3D images was accomplished using Imaris (Bitplane, South Windsor, CT, USA) version 6.2. Isosurface renderings of capillaries in each data stack were made using Imaris software (Bitplane) and vessel volume determined with Imaris Measurement Pro. The total vessel volume in each sample was divided by the total tissue volume for the vascular density in each sample.

\section{Quantitative real-time PCR}

Total RNA was extracted from the epididymal adipose tissue using a TRIzol reagent (Invitrogen). cDNA was synthesized using the Prime Script RT Reagent Kit (Takara Bio). Real-time qPCR was performed on the LightCycler ${ }^{\circledR} 480$ System with LightCycle® 480 SYBR Green Master (04897030001, Roche). Fold change of mRNA expression was determined using the $2^{-\Delta \Delta C T}$ method, with all genes normalized to the mouse $\beta$-actin. All qPCR primers and their sequence in this study are presented in Table 1.
Table 1 Sequence of primers used for quantitative real-time PCR.

\begin{tabular}{|c|c|}
\hline Primers & DNA sequences \\
\hline II-6 F & 5'-CCGGAGAGGAGACTTCACAG-3' \\
\hline II-6 R & 5'-ACAGTGCATCATCGCTGTTC-3' \\
\hline$\| l-1 b \mathrm{~F}$ & 5'-GCAACTGTTCCTGAACTCA-3' \\
\hline$\| l-1 b \mathrm{R}$ & 5'-CTCGGAGCCTGTAGTGCAG-3' \\
\hline$M c p-1 \mathrm{~F}$ & 5'-TGTTCCTCTTAATCCTGCCCA-3' \\
\hline Mcp-1 R & 5'-TCTGGACCCATTCCTTCTTG-3' \\
\hline F4/80 F & 5'-GGAAAGCACCATGTTAGCTGC-3' \\
\hline F4/80 R & 5'-CCTCTGGCTGCCAAGTTAATG-3' \\
\hline Hif-1a F & 5'-GATGAGTTCTGAACGTCGAAAAGAAAAGT-3' \\
\hline Hif-1a R & 5'-GAAGTTTTCTCACACGTAAATAACTGATGGTG-3' \\
\hline Vegf $\mathrm{F}$ & 5'-GAGCAGAAGTCCCATGAAGTGA-3' \\
\hline Vegf $\mathrm{R}$ & 5'-CACAGGA CGGCTTGAAGATGT-3' \\
\hline Vegfr2 F & 5'-GCTCCGCCCCCAACTCCT-3' \\
\hline Vegfr2 R & 5'-CTCATAGCCCGCATCCTCCACA-3' \\
\hline Et-1 $\mathrm{F}$ & 5'-TGTCTTGGGAGCCGAACTCA-3' \\
\hline Et-1 R & 5'-GCTCGGTTGTGCGTCAACTTCTGG-3' \\
\hline Pdgfa F & 5'-CAGTGTCAAGGTGGCCAAAGT-3' \\
\hline Pegfa R & 5'-TGGTCTGGGTTCAGGTTGGA-3' \\
\hline Pegfb F & 5'-TAATGTTGTTCCCCTCGTCC-3' \\
\hline Pegfb R & 5'-AAGGCTCCTGCACACTTGTT-3' \\
\hline$T g f b \mathrm{~F}$ & 5'-CGAAGCGGACTACTATGCTAAAGAG-3' \\
\hline Tgfb R & 5'-CGTCAAAAGACAGCCACTCAGG-3' \\
\hline Pedf F & 5'-GTGGGCAACCAAGTTTGACT-3' \\
\hline Pedf R & 5'-AGGGGCAGGAAGAAGATGAT-3' \\
\hline $\mathrm{Cd} 31 \mathrm{~F}$ & 5'-AGCCAACAGCCATTACGGTTA-3' \\
\hline $\mathrm{Cd} 31 \mathrm{R}$ & 5'-AGCCTTCCGTTCTCTTGGTG-3' \\
\hline Ve-cad F & 5'-ATTGGCCTGTGTTTTCGCAC-3' \\
\hline Ve-cad R & 5'-CACAGTGGGGTCATCTGCAT-3' \\
\hline Angpt1 $\mathrm{F}$ & 5'-ACATGGGCAATGTGCCTACA-3' \\
\hline Angpt1 $\mathrm{R}$ & 5'-TTTGCAGAGCGTTGGTGTTG-3' \\
\hline Angpt2 F & 5'-AGTACCAGGTCCAGAACGGA-3' \\
\hline Angpt2 R & 5'-ACGGCATTGGACATGTAGGG-3' \\
\hline Fgf1 F & 5'-TTATACGGCTCGCAGACACC-3' \\
\hline Fgf1 R & 5'-TCTCCGCATGCTTCTTGGAG-3' \\
\hline Fgf $2 \mathrm{~F}$ & 5'-GCTGCTGGCTTCTAAGTGTG-3' \\
\hline Fgf2 R & 5'-ACTGCCCAGTTCGTTTCAGT-3' \\
\hline Fgf10 F & 5'-GCATGTGCGGAGCTACAATC-3' \\
\hline Fgf10 R & 5'-TTCATTCTTGGTCCCGCTGA-3' \\
\hline Glut1 F & 5'-ATGGATCCCAGCAGCAAGAAG-3' \\
\hline Glut1 R & 5'-CCAGTGTTATAGCCGAACTGC-3' \\
\hline Pdk1 F & 5'-TGCAAAGTTGGTATATCCAAAGCC-3' \\
\hline Pdk1 R & 5'-TGTGCCGGTTTCTGATCCTT-3' \\
\hline Gpx1 F & 5'-AATGTCGCGTCTCTCTGAGG-3' \\
\hline Gpx1 R & 5'-TTGCCATTCTGGTGTCCGAA-3' \\
\hline$V h / F$ & 5'-GACTACCCAAGTGTGCGGAA-3' \\
\hline$V h / \mathrm{R}$ & 5'-CAAGGCTCCTCTTCCAGGTG-3' \\
\hline Adam8 F & 5'-TAGCCCCTGGACCTCCTTTG-3' \\
\hline Adam8 R & 5'-CGCTGGTCCCAAGAGCATAG-3' \\
\hline$\beta$-actin $\mathrm{F}$ & 5'-GCCCTGAGGCTCTTTTCCAG-3' \\
\hline$\beta$-actin R & 5'-GCTGGAAGGTGGACAGTGAG-3' \\
\hline
\end{tabular}

\section{Western blotting}

Methods for protein extraction and Western blotting have been described previously ( $\mathrm{Xu}$ et al. 2016). Antibodies against vascular endothelial growth factor (VEGF, sc-507), vascular endothelial growth factor receptor 2 
(VEGFR2, sc-504), platelet/endothelial cell adhesion molecule 1 (PECAM-1, sc-28188), insulin receptor substrate-1 (IRS1, sc-7200) were purchased from Santa Cruz Biotechnology. Antibodies against insulin receptor beta (IRbeta, CST\#3025), phosphorylation of IRS1 (p-IRS1, CST\#3070), Akt kinase (Akt, CST\#4691), phosphorylation of Akt (p-Akt, CST\#4060), phosphoinositide-3-kinase (PI3K, CST\#4249) were purchased from Cell Signaling Technology. Beta-actin (CST\#9470, Cell Signaling Technology) served as a loading control.

\section{Immunohistochemistry}

The epididymal fat pads were isolated and fixed in neutral buffered formalin, then dehydrated, and embedded in paraffin. Thin tissue slides $(5 \mu \mathrm{m})$ were deparaffinized, blocked and incubated overnight at $4^{\circ} \mathrm{C}$ with a rat antimouse F4/80 (sc-71085, Santa Cruz Biotechnology), a mouse anti-mouse hypoxia-inducible factor (HIF)-1alpha (NB100-105, Novus Biologicals, Littleton, CO, USA) and a rabbit anti-mouse PECAM-1 antibody (ab28364, Abcam), which was followed by signal amplification using a VECTASTAIN Elite ABC Kit (PK-6102; Vector Laboratories). The reaction was developed by addition of AEC chromogen substrate (AEC Staining kit; SigmaAldrich). All sections were mounted and analyzed at 100× and 400× magnifications using a digitalized LEICA DMi8 microscope. Three to five random microscopic fields were selected in each sample. The expression of F4/80, HIF-1alpha and PECAM-1 were analyzed and evaluated by the average optical density (AOD), as measured by Image J software.

\section{Statistical analysis}

All data are expressed as means \pm s.E. Results were analyzed by student t-test or ANOVA and statistical significance was set at $P<0.05$.

\section{Results}

\section{Diet induced obesity and impaired glucose homeostasis in mice are improved by exenatide}

Male C57BL/6J mice were fed with HFD for 12 weeks to develop obesity. As shown, body weight (Fig. 1A), fat mass (Fig. 1B and C) and ratio of fat mass to body weight (Fig. 1D) in HFD-fed mice were significantly higher when compared with NC mice. After a 4-week exenatide treatment, HFD-challenged mice exhibited significant weight loss and reduced fat mass (Fig. 1A, B, C and D), which were associated with reduced food intake (Fig. 1E). The fasting blood glucose (FBG) level of the HFDchallenged mice was higher than the level of those fed on the chow diet, and presented a significant reduction after exenatide treatment (Fig. 1F). The i.p.GTT and i.p.ITT were conducted after 4 weeks of exenatide administration. The result of i.p.GTT showed that HFD-challenged mice had impaired glucose intolerance, as indicated by the higher glucose levels at all the time points during the test and the significantly larger area under the curve (AUC) value (Fig. 1G). During the i.p.ITT, higher blood glucose levels at time points 90 and $120 \mathrm{~min}$ as well as a larger AUC value were observed in HFD-challenged mice (Fig. $1 \mathrm{H}$ ). After exenatide treatment, the deteriorated glucose tolerance and insulin sensitivity of the DIO mice were both improved (Fig. $1 \mathrm{G}$ and $\mathrm{H}$ ).

\section{Exenatide suppresses obesity associated pro-inflammatory cytokine production and macrophage infiltration in adipose tissue}

To examine the diet-induced adipose tissue inflammation, pro-inflammatory factors including IL-6, PAI and TNFalpha were measured in serum. Levels of these cytokines were higher in HFD group compared to NC group (Fig. 2A, $B$ and $C)$. And all of them were significantly reduced by exenatide treatment. Meanwhile, relative mRNA levels of $I l-6$ and $I l-1 b$ in epididymal fat of HFD-challenged mice were obviously up-regulated in comparison to NC group (Fig. 2D). However, exenatide treatment significantly down-regulated mRNA expression of these two inflammatory cytokines (Fig. 2D). Since accumulating studies showed that the elevated inflammatory status appeared to originate from infiltrated macrophages, we also tested the mRNA expression of $M c p-1$, a member of the small inducible chemokine family that played a role in the recruitment of monocytes to sites of injury and infection; and F4/80, a classical macrophage-restricted surface. The relative mRNA level of $M c p-1$ in the adipose tissues was drastically increased in HFD mice compared with NC mice, whereas exenatide significantly decreased its expressions (Fig. 2D). Quantitative RT-PCR and immunohistochemistry measurements showed that the mRNA and protein expression of F4/80 in epididymal fat tissues were significantly increased in HFD-challenged mice compared with NC group (Fig. 2D and E), and the protein level of F4/80 was downregulated by exenatide treatment (Fig. 2E and F). Together, these data indicate 
A
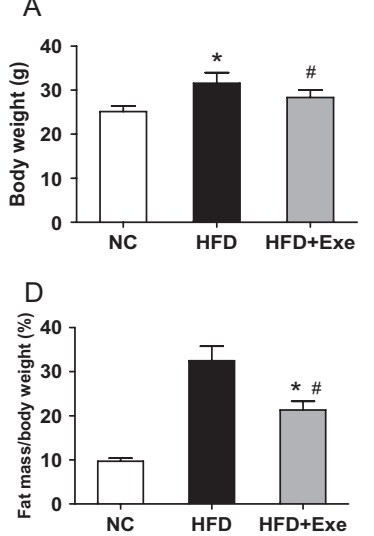

G

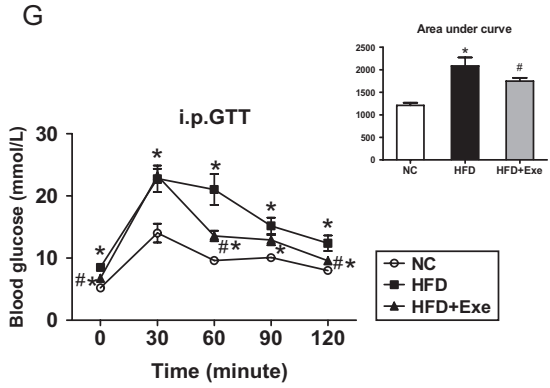

$\mathrm{H}$

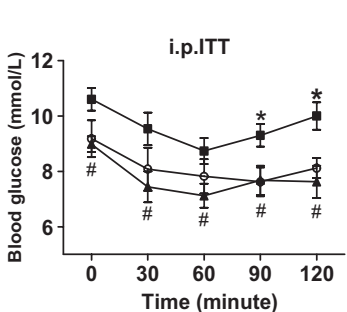

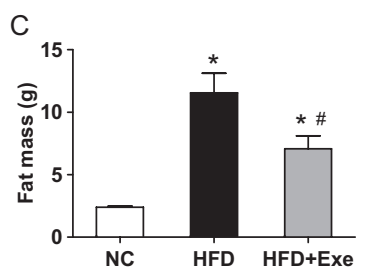

$\mathrm{F}$
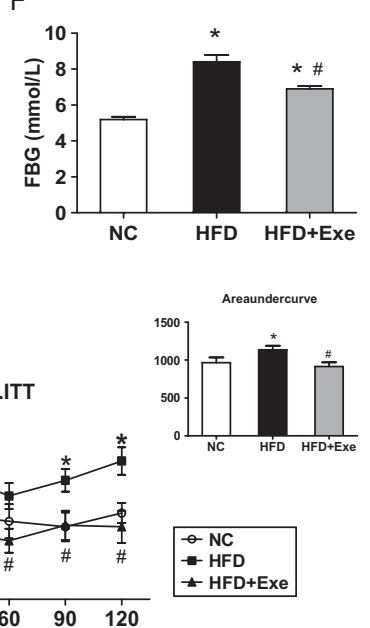

Figure 1

Diet induced obesity and impaired glucose homeostasis in mice are improved by exenatide. Mice in the HFD group were randomized to receive exenatide or normal saline for 4 weeks after a 12-week HFD challenge. (A) Body weight, (B) epididymal fat weight, (C) fat mass, (D) fat mass/body weight, (E) food intake, (F) FBG were analyzed among three groups. The fat percentage in the body composition was determined by the ratio of fat mass to body weight. i.p.GTT (G) and i.p.ITT $(H)$ were performed at the end of the intervention with exenatide or normal saline treatment. The area under the curve (AUC) was determined. $n=5$ mice per group. All values are expressed as the mean \pm S.E.M. ${ }^{*} p<0.05$ when compared with NC group; $\# p<0.05$ when compared with HFD group. that obesity is accompanied with chronic low-grade inflammation, and exenatide can broadly suppress proinflammatory cytokines in serum and adipose tissue and reduce macrophage infiltration in adipose tissue.

\section{Exenatide alleviates hypoxia in adipose tissue of HFD-challenged mice}

It has been widely accepted that hypoxia resulted in inflammation in adipose tissue (Ye et al. 2007). To clarify the underlying mechanism of exenatideameliorated inflammation, we detected protein levels of HIF-1alpha in epididymal fat of C57BL/6 mice. As shown by immunohistochemistry staining, HIF-1alpha expression was higher in HFD mice than NC group (Fig. 3A and B), supporting the hypothesis that hypoxia occurred in adipose tissue with obese state. Exenatide obviously reduced the protein level of HIF-1alpha in HFD-challenged mice when compared with HFD group (Fig. 3A and B). Similarly, mRNA levels of genes involved in hypoxia pathway, including Glut1, Pdk1, Gpx1, Vhl and Adam 8 were significantly higher in HFD group than that in NC group (Fig. 3C). After exenatide treatment, the mRNA levels of Hif-1a, Pdk1, Vhl and Adam8 were drastically decreased (Fig. 3C). These results indicate that hypoxia in fat tissue of obese status can be reversed by exenatide.
Exenatide promotes angiogenesis and improves insulin sensitivity in adipose tissue of HFD-challenged mice

We further detected the expression of angiogenic growth factors in adipose tissue. As shown, the protein levels of pro-angiogenic factors such as VEGF and VEGFR2 in epididymal fat of obese C57BL/6J mice were significantly increased when compared with NC group (Fig. 4A, B and C). Similarly, the mRNA levels of pro-angiogenic factors including endothelin 1 (Et-1), platelet-derived growth factor alpha ( $P d g f a)$, platelet-derived growth factor beta $(P d g f b)$ and transforming growth factor beta $(T g f b)$ in adipose tissue of HFD group were up-regulated in the mRNA levels (Fig. 4D) when compared with NC group. These results suggested that angiogenic factors were upregulated in response to hypoxia in obesity. After exenatide treatment, the protein levels of VEGF and VEGFR2 were further elevated in obese mice (Fig. 4A, $B$ and $C)$. Accordingly, the mRNA levels of majority of pro-angiogenic factors, including Vegf, Vegfr2, Pdgfb, angiopoietin 1 (Angpt1), Angpt2, fibroblast growth factor $(F g f 1), F g f 2$ and $F g f 10$ were drastically upregulated by exenatide in obese mice, while the mRNA level of platelet derived growth factor (Pedf), an anti-angiogenic factor, tended to be downregulated in HFD-challenged mice (Fig. 4D). These results indicate that angiogenic factors mainly including VEGF and VEGFR2 are promoted by 
A

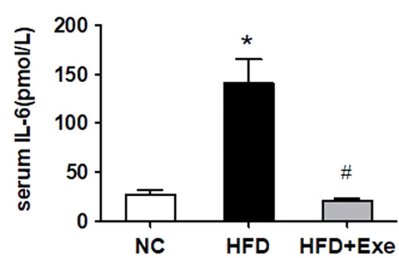

B

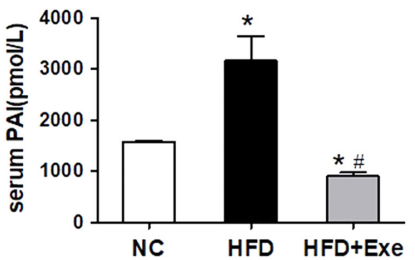

C

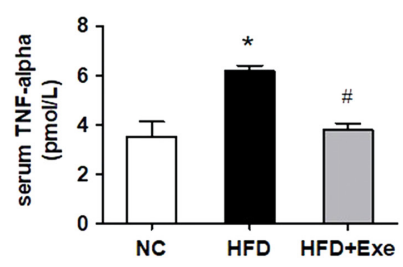

D

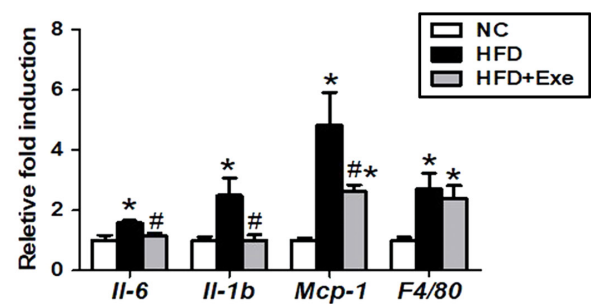

E

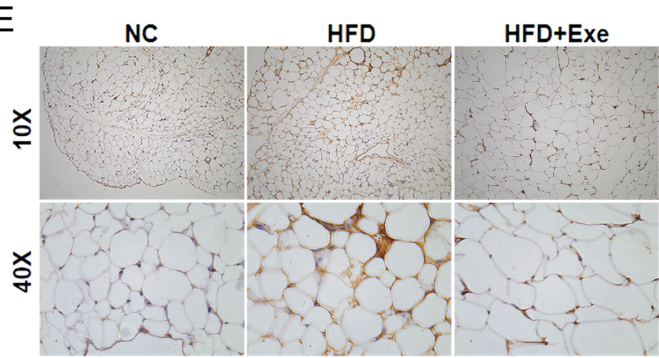

$\mathrm{F}$

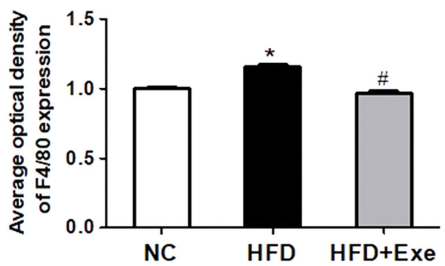

Figure 2

Exenatide suppresses obesity associated pro-inflammatory cytokine production and macrophage infiltration in adipose tissue. After 4 weeks exenatide treatment, C57BL/6J mice were killed and blood samples and epididymal fat tissues were collected. Several key proinflammatory factors were measured by ELISA and real-time qPCR. (A, B and C) Serum levels of IL-6, PAI and TNF-alpha in epididymal fat tissues. (D) Relative mRNA levels of II-6, II-1 $b, M c p-1$ and F4/80 were tested in epididymal fat tissue respectively. (E) F4/80 immunohistochemistry detecting murine macrophage infiltration in epididymal fat tissue. The upper line shows microphotographs taken under an objective lens $(10 \times)$ and the lower line shows those taken under an objective lens (40x). (F) Quantitative data of F4/80 expression measured by immunohistochemistry. $n=3-5$ samples per group. All values are expressed as the mean \pm S.E.M. ${ }^{*} p<0.05$ when compared with NC group; $\# p<0.05$ when compared with HFD group. exenatide in adipose tissue of DIO mice. We also measured protein level and phosphorylation status of several molecules involved in the insulin signaling pathway. As expected, exenatide significantly upregulated the protein expression of IR, phosphorylation of IRS-1 and AKT, PI3K in adipose tissue of DIO mice (Fig. 4E and F), indicating that insulin signaling pathway was activated. Taken together, we conclude that exenatide helps to regenerate microcirculation, which may contribute to the reversed hypoxia and improved insulin sensitivity in obese fat tissue.

\section{Exenatide ameliorates the impaired microvasculature in adipose tissue of HFD-challenged mice}

In order to investigate whether exenatide improved microvasculature of adipose tissue, we conducted vascular imaging in epididymal fat. The images showed that the size of adipocytes in DIO C57BL/6J mice dramatically increased and capillary volume decreased when compared with NC mice (upper, Fig. 5A). This indicated that microvasculature was deficient in the adipose tissue of obese mice. After 4-week exenatide treatment, microvasculature was markedly induced in HFD-challenged mice when compared with those receiving saline injection (upper, Fig. 5A). A clean capillary network was generated and used to calculate the vascular volume (below, Fig. 5A). Vascular volume was then divided by the adipocytes and total adipose tissue volume for normalization respectively. Consistent with the confocal imaging, the quantitation of vessels showed that capillary density in the epididymal fat of HFD-challenged group was significantly elevated by exenatide (Fig. 5B and C). Furthermore, the capillary was determined by an endothelial cell marker, PECAM-1, in epididymal fat. Western blotting and the corresponding densitometric analysis showed that exenatide augmented protein levels of PECAM-1 in epididymal fat of HFDchallenged mice in comparison with those with saline treatment (Fig. 5D). In accordance with this result, immunohistochemistry analysis showed that, PECAM-1, as distributed in the extracellular matrix of adipocytes, was obviously elevated by exenatide treatment (Fig. 5E and F). All these data indicate that despite the elevated expression of pro-angiogenic factors in adipose tissue of DIO mice, the ability of vasculature construction of them is restricted. However, this restriction can be ameliorated by exenatide.

\section{Discussion}

Chronic low-grade inflammation and macrophage infiltration in adipose tissue have been reported to be 
A

A NC

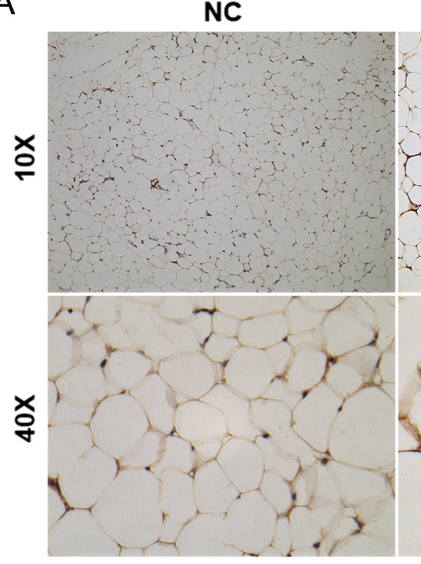

B

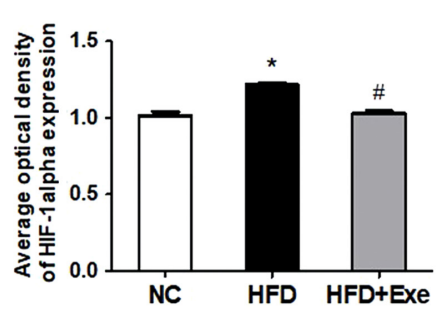

HFD

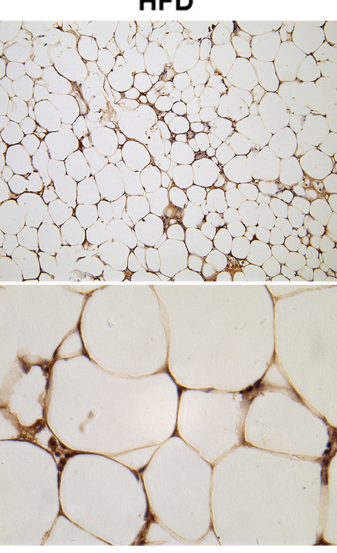

HFD+Exe

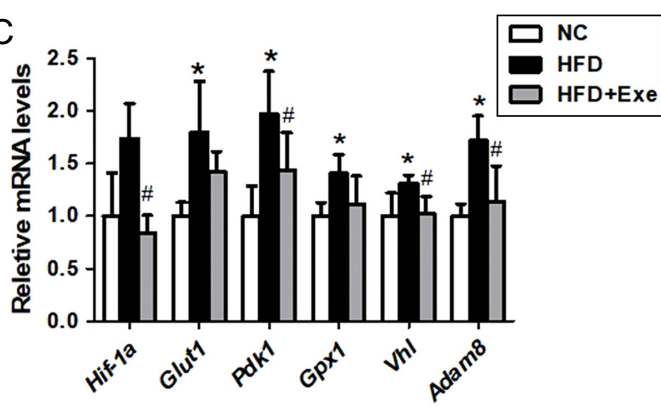

\section{Figure 3}

Exenatide alleviates hypoxia in adipose tissue of HFD-challenged mice. Total RNA was extracted from the epididymal fat and examined for gene expression. (A) Immunohistochemical detection of HIF-1alpha in epididymal fat tissue. The upper line shows microphotographs taken under an objective lens (10x) and the lower line shows those taken under an objective lens (40x). (B) Quantitative data of HIF-1alpha expression measured by immunohistochemistry. (C) Real-time qPCR was used to quantify mRNA for hypoxia response genes as Hif-1a, Glut1, Pdk1, Gpx1, Vhl, and Adam8. $n=3-5$ samples per group. All values are expressed as the mean \pm S.E.M. ${ }^{*} p<0.05$ when compared with NC group; ${ }^{*} p<0.05$ when compared with HFD group. closely related to the development of insulin resistance in obesity (de Heredia et al. 2012). In the current study, DIO mice manifest higher levels of circulating inflammatory cytokines and their corresponding increased mRNA expressions in adipose tissue including MCP-1, which promotes macrophage infiltration into adipose tissue (Kanda et al. 2006). The increase of macrophage infiltration in adipose tissue, which largely correlates with the degree of obesity, is responsible for producing a significantly elevated level of inflammatory cytokines (Weisberg et al. 2003). Moreover, our study confirms the existence of hypoxia in adipose tissue under obesity state. It is well-established that hypoxia could induce proinflammatory gene expressions (e.g. TNF-alpha, MCP-1) by activating HIF-1alpha (Ye et al. 2007). Deletion of HIF1alpha in adipocytes reduces macrophage infiltration and inflammation in adipose tissue (Kihira et al. 2014). HIF-1alpha is also involved in the induction of proinflammatory M1-like polarity of macrophages (Fujisaka et al. 2013) and the formation of crown-like structures in adipose tissue (Takikawa et al. 2016), further enhancing the inflammatory response in obesity.

Regarding to the cause of hypoxia, it is putatively suggested that as adipose tissue mass expands in obesity, clusters of enlarged adipocytes become distant from the vasculature, leading to local pockets of hypoxia (Trayhurn $\&$ Wood 2004). As a counter regulatory response to

(C) 2019 Society for Endocrinology Published by Bioscientifica Ltd.
Printed in Great Britain hypoxia during its expansion, adipose tissue expresses various angiogenic growth factors, such as VEGF, for neovascularization (Wang et al. 2007). Despite of the increased pro-angiogenic factors in adipose tissue, the ability of vasculature construction is hampered in obesity, as shown by the HFD induced reduction in capillary density and endothelial cells in our study. It is consistent with previous studies, which have found that capillary density and endothelial cells function failed to meet the demand of adipose tissue growth in obesity (Pang et al. 2008, Spencer et al. 2011). This failure leads to an impaired blood perfusion and thus microcirculation dysfunction (Hosogai et al. 2007, Pasarica et al. 2009), which aggravates a local hypoxia in adipose tissue. Recent study also showed that the HIF-1a knock out DIO mice exhibited improved glucose metabolism with less inflammation, less hypoxia and better vasculature development in adipose tissue (Takikawa et al. 2016). This indicates that hypoxia inhibits vasculature development in adipose tissue in HFD-induced obesity.

In addition to weight loss and glucose-lowering effects, emerging data suggests that GLP-1-based therapies show anti-inflammatory effects in chronic inflammatory diseases including obesity (Lee \& Jun 2016). It was proved that administration of a recombinant adenovirus producing GLP-1 to ob/ob mice reduced the macrophage population and production of TNF-alpha, MCP-1 and 
A
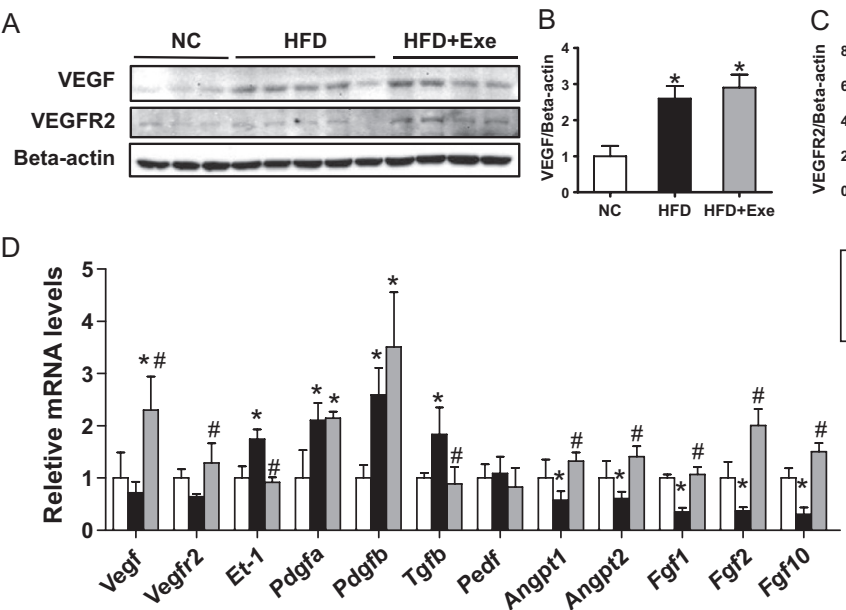

E
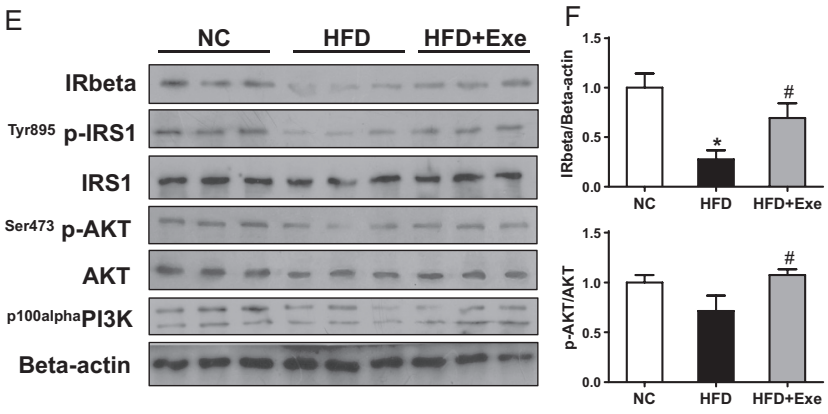

IL-6 in adipose tissue through inhibiting nuclear factor$\kappa \mathrm{B}$ pathway (Lee et al. 2012). Similarly, the present study shows that exenatide treatment results in a significant reduction in plasma levels of various inflammatory markers and macrophage infiltration in adipose tissue of DIO mice. In addition, obesity associated hypoxia is reversed by exenatide, which may contribute to the reduced inflammatory mediators and the improved insulin sensitivity in adipose tissue.

Our study also reveals that the impairment in neovascularization of obese adipose tissue is alleviated by exenatide, as shown by increased capillary density and endothelial cell marker PECAM-1. Previous study showed that GLP-1 exerted beneficial effects on endothelial cell independently of its action on glucose, lipid or energy metabolism in subjects with T2D (Goyal et al. 2010). For example, exenatide stimulated proliferation of human coronary artery endothelial cells through eNOS-, PKAand PI3K/Akt-dependent pathway via a GLP-1 receptordependent mechanism (Erdogdu et al. 2010). Besides, exenatide directly improved endothelial dysfunction in isolated aortas from obese rats through the cAMP or AMPK-eNOS pathways (Han et al. 2012). The important role of exendin- 4 in modulating angiogenesis was also supported by the fact that exenatide directly regulated
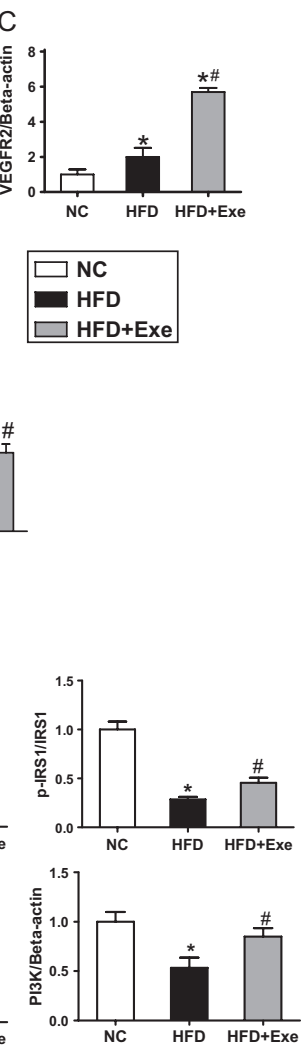

\begin{abstract}
Figure 4
Exenatide promotes angiogenesis and improves insulin sensitivity in adipose tissue of HFDchallenged mice. Protein expression levels of pro-angiogenic factors and molecules involving the insulin signaling pathway in epididymal fat tissues were measured by Western blotting. Key genes related to angiogenesis were detected by real-time QPCR in epididymal fat tissues. (A, B and C) Protein levels of VEGF and VEGFR2 and densitometric analyses of these panels. Beta-actin, loading control. (D) Relative mRNA levels of Vegf, Vegfr2, Et-1, Pdgfa, Pdgfb, Tgfb, Pedf, Angpt1, Angpt2, Fgf1, Fgf2 and Fgf10 were tested in epididymal fat tissue respectively. ( $E$ and $F$ ) Protein levels of insulin signaling pathway (IRbeta, p-IRS1, IRS1, p-Akt, Akt, PI3K) and densitometric analyses of these panels. Beta-actin, loading control. $n=3-4$ samples per group. All values are expressed as the mean \pm S.E.M. ${ }^{*} p<0.05$ when compared with NC group; $\# p<0.05$ when compared with HFD group.
\end{abstract}

proliferation, migration, spouting and tube formation of endothelial cells, thus induced neovascularization (Kang et al. 2013). GLP-1 was also reported to be able to protect microvascular endothelial cells against apoptosis by inactivating the PARP-1/iNOS/NO pathway (Liu etal. 2011). Recent studies showed that GLP-1 receptor activation could mitigate vascular endothelial senescence (Lei et al. 2017, Yang et al. 2017) and restore impaired ischemiainduced blood flow recovery and capillary formation in stressed aortas and muscle (Piao et al. 2017). This may be partially attributable to the reduction of inflammation and enhancement of adiponectin production (Piao et al. 2017, Yang et al. 2017). All these studies indicate that GLP-1 ameliorates endothelial dysfunction, which might promote angiogenesis and improve microcirculation to provide sufficient blood flow and oxygen for the expanding adipose tissue in obesity. Our result shows that exenatide significantly restored capillary density and down-regulated HIF-1alpha in obese mice. It indicates that exenatide might improve microcirculation and thus alleviate hypoxia in the adipose tissue.

On molecular mechanism, the beneficial effect of exenatide on angiogenesis is shown by the upregulation of pro-angiogenic factor VEGF and its receptor VEGFR2 in adipose tissue in the current study. VEGF exerts its 
A

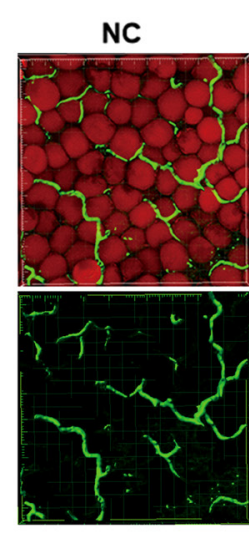

E

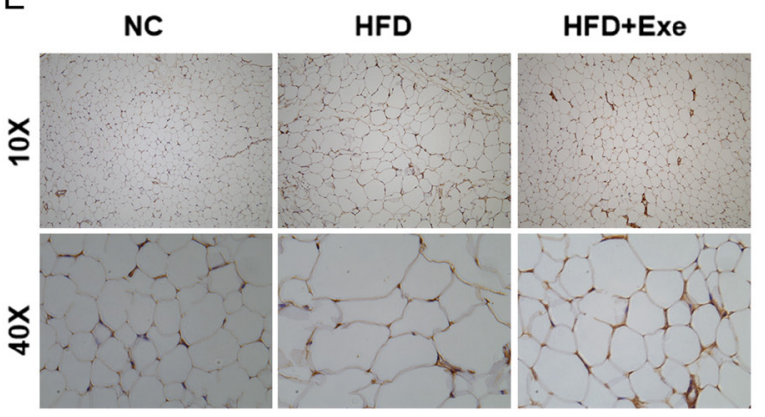

B

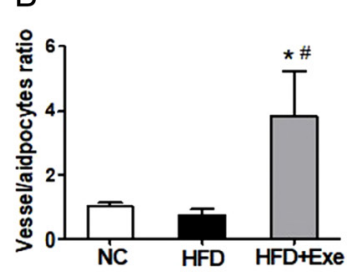

C
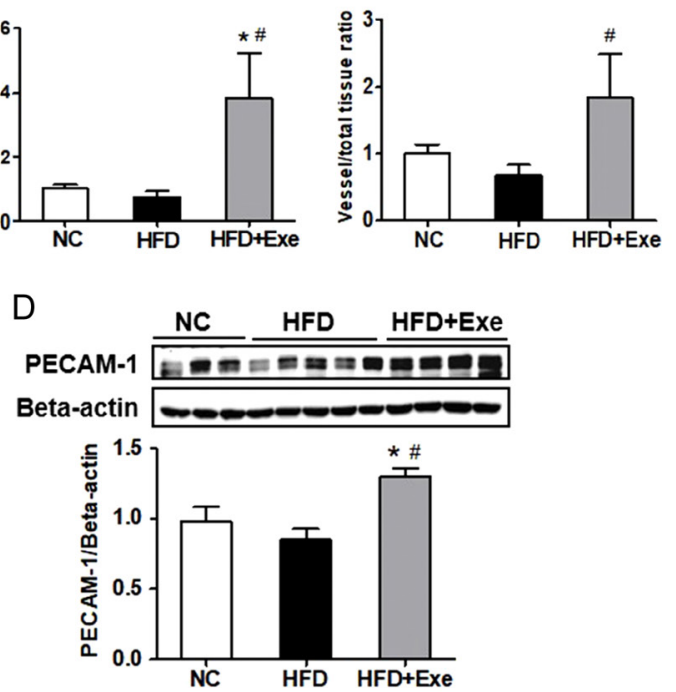

F

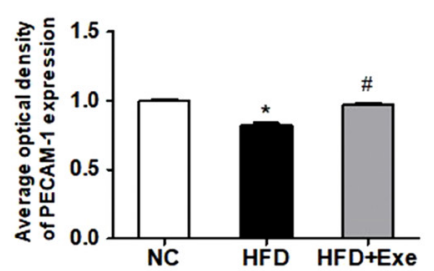

\section{Figure 5}

Exenatide ameliorates the impaired microvasculature in adipose tissue of HFD-challenged mice. Small pieces of epididymal fat tissues were stained with GS-IB4 and BODIPY overnight for blood vessels and adipocytes measurement respectively. Vessels volume and adipocytes volume were determined by confocal microscopy and 3D reconstruction. (A) 3D images of epididymal fat tissues with adipocytes stained in red and vessels in green, as shown in the upper line. After exclusion of adipocytes, capillary density was displayed in the lower line. (B) Vessels/adipocytes volume ratio. (C) Vessels/ total tissue volume ratio. (D) Protein levels and densitometric analyses of PECAM-1 Beta-actin, loading control. (E) Immunohistochemical detection of endothelial marker PECAM-1 in epididymal fat tissue. The upper line shows microphotographs taken under an objective lens (10x) and the lower line shows those taken under an objective lens (40×). (F) Quantitative data of PECAM-1 expression measured by immunohistochemistry. $n=3-5$ samples per group. All values are expressed as the mean \pm S.E.M. ${ }^{*} p<0.05$ when compared with NC group; ${ }^{*} p<0.05$ when compared with HFD group.

pro-angiogenic action by binding to VEGFR2, a process that is required for adipocyte differentiation and adipose tissue growth (Kinugasa et al. 2012). Previous study revealed that GLP-1 markedly enhanced the proliferation of circulating endothelial progenitor cells derived from bone marrow through increasing VEGF generation (Xie et al. 2011). Moreover, GLP-1 promoted angiogenesis in human endothelial cells in a dose-dependent manner through the Akt and Src pathways (Aronis et al. 2013), both of which had a pivotal role in VEGF-induced angiogenesis (Bir et al. 2012). Therefore, VEGF might be a downstream target of GLP-1 and GLP-1 receptor agonist in regulating angiogenesis.

Previous studies showed that GLP-1 infusion potently expanded muscle microvascular surface area and improves insulin resistance (Chai et al. 2012, 2014). Our current study find that GLP-1 receptor activation-mediated augmentation of adipose angiogenesis is accompanied with reversed hypoxia, reduced inflammation in obese adipose tissue, which may contribute to the improvement of insulin sensitivity in white adipose tissue. It will be interesting to examine whether there is any causal effect among these factors. In addition, previous study found that macrophage HIF-1alpha negatively regulated vessel formation by suppressing the expression of angiogenic factors, including VEGF, not only in preadipocytes, but also in endothelial cells and adipocytes (Takikawa et al. 2016). It will be worth investigating whether exenatide directly acts on macrophage or other cells in obese adipose tissue for the upregulation of angiogenic factors and the improvement of hypoxia.

In conclusion, our study demonstrates that obesity is accompanied with inflammation, and hypoxia along with insufficient neovascularization in adipose tissue is a potential risk factor for this chronic inflammation. Most importantly, GLP-1 receptor agonist exenatide 
protects against obesity-related inflammation and hypoxia in adipose tissue, which may contribute to a local improvement of insulin resistance. Moreover, this might be associated with exenatide's positive effect on angiogenesis and microcirculation in adipose tissue. Further research is deserved to investigate how GLP-1 regulates angiogenesis and microcirculation, and whether there is any causal effect among these factors.

\section{Declaration of interest}

The authors declare that there is no conflict of interest that could be perceived as prejudicing the impartiality of the research reported.

\section{Funding}

This study was supported by the National Natural Science Foundation of China Grant (81670782 to FX), Pearl River S\&T Nova Program of Guangzhou (201610010175 to F X), Guangdong High-Level Talents Special Support Program (2016TQ03R590 to F X), Local Innovative and Research Teams Projects of Guangdong Pearl River Talents Program (2017BT 01S131 to J W and F X), National Natural Science Foundation of China Grant (81770821 to WX, 81670762 to $M$ C).

\section{Author contribution statement}

$\mathrm{Y} X$ contributed to the immunochemistry analysis, interpretation and discussion of data, and writing and revising of the manuscript. Z C researched data, performed animal studies, analyzed data and wrote the manuscript. HD analyzed data. M C, H L and WX contributed to the revision of the manuscript. J $W$ contributed to the study design, revision of the manuscript, and approval of the version to be submitted. $\mathrm{F} X$ contributed to the study design, acquisition of data, data interpretation, manuscript writing and revising and approval of the version to be submitted. $\mathrm{FX}$ is the guarantor of this work and, as such, had full access to all the data in the study and takes responsibility for the integrity of the data and the accuracy of the data analysis.

\section{Acknowledgments}

The authors thank Z Li and B Lin for their assistance with the animal experiments.

\section{References}

Aronis KN, Chamberland JP \& Mantzoros CS 2013 GLP-1 promotes angiogenesis in human endothelial cells in a dose-dependent manner, through the Akt, Src and PKC pathways. Metabolism 62 1279-1286. (https://doi.org/10.1016/j.metabol.2013.04.010)

Bir SC, Xiong Y, Kevil CG \& Luo J 2012 Emerging role of PKA/eNOS pathway in therapeutic angiogenesis for ischaemic tissue diseases. Cardiovascular Research 95 7-18. (https://doi.org/10.1093/cvr/cvs143)

Chai W, Dong Z, Wang N, Wang W, Tao L, Cao W \& Liu Z 2012 Glucagon-like peptide 1 recruits microvasculature and increases glucose use in muscle via a nitric oxide-dependent mechanism. Diabetes 61 888-896. (https://doi.org/10.2337/db11-1073)
Chai W, Zhang X, Barrett EJ \& Liu Z 2014 Glucagon-like peptide 1 recruits muscle microvasculature and improves insulin's metabolic action in the presence of insulin resistance. Diabetes 63 2788-2799. (https://doi.org/10.2337/db13-1597)

Chaudhuri A, Ghanim H, Vora M, Sia CL, Korzeniewski K, Dhindsa S, Makdissi A \& Dandona P 2012 Exenatide exerts a potent antiinflammatory effect. Journal of Clinical Endocrinology and Metabolism 97 198-207. (https://doi.org/10.1210/jc.2011-1508)

Christiaens V \& Lijnen HR 2010 Angiogenesis and development of adipose tissue. Molecular and Cellular Endocrinology 318 2-9. (https:// doi.org/10.1016/j.mce.2009.08.006)

de Heredia FP, Gomez-Martinez S \& Marcos A 2012 Obesity, inflammation and the immune system. Proceedings of the Nutrition Society $\mathbf{7 1}$ 332-338. (https://doi.org/10.1017/S0029665112000092)

Donath MY \& Shoelson SE 2011 Type 2 diabetes as an inflammatory disease. Nature Reviews Immunology 11 98-107. (https://doi. org/10.1038/nri2925)

Erdogdu Ö, Nathanson D, Sjoholm Å, Nystrom T \& Zhang Q 2010 Exendin-4 stimulates proliferation of human coronary artery endothelial cells through eNOS-, PKA- and PI3K/Akt-dependent pathways and requires GLP-1 receptor. Molecular and Cellular Endocrinology 325 26-35. (https://doi.org/10.1016/j.mce.2010.04.022)

Farb MG, Ganley-Leal L, Mott M, Liang Y, Ercan B, Widlansky ME, Bigornia SJ, Fiscale AJ, Apovian CM, Carmine B, et al. 2012 Arteriolar function in visceral adipose tissue is impaired in human obesity. Arteriosclerosis, Thrombosis, and Vascular Biology 32 467-473. (https:// doi.org/10.1161/ATVBAHA.111.235846)

Fujisaka S, Usui I, Ikutani M, Aminuddin A, Takikawa A, Tsuneyama K, Mahmood A, Goda N, Nagai Y, Takatsu K, et al. 2013 Adipose tissue hypoxia induces inflammatory M1 polarity of macrophages in an HIF-1alpha-dependent and HIF-1alpha-independent manner in obese mice. Diabetologia 56 1403-1412. (https://doi.org/10.1007/s00125013-2885-1)

Gealekman O, Guseva N, Hartigan C, Apotheker S, Gorgoglione M, Gurav K, Tran KV, Straubhaar J, Nicoloro S, Czech MP, et al. 2011 Depot-specific differences and insufficient subcutaneous adipose tissue angiogenesis in human obesity. Circulation 123 186-194. (https://doi.org/10.1161/CIRCULATIONAHA.110.970145)

Goyal S, Kumar S, Bijjem KV \& Singh M 2010 Role of glucagon-like peptide-1 in vascular endothelial dysfunction. Indian Journal of Experimental Biology 48 61-69.

Han L, Yu Y, Sun X \& Wang B 2012 Exendin-4 directly improves endothelial dysfunction in isolated aortas from obese rats through the cAMP or AMPK-eNOS pathways. Diabetes Research and Clinical Practice 97 453-460. (https://doi.org/10.1016/j.diabres.2012.04.001)

Hosogai N, Fukuhara A, Oshima K, Miyata Y, Tanaka S, Segawa K, Furukawa S, Tochino Y, Komuro R, Matsuda M, et al. 2007 Adipose tissue hypoxia in obesity and its impact on adipocytokine dysregulation. Diabetes 56 901-911. (https://doi.org/10.2337/db060911)

Kanda H, Tateya S, Tamori Y, Kotani K, Hiasa K, Kitazawa R, Kitazawa S, Miyachi H, Maeda S, Egashira K 2006 MCP-1 contributes to macrophage infiltration into adipose tissue, insulin resistance, and hepatic steatosis in obesity. Journal of Clinical Investigation 116 1494-1505. (https://doi.org/10.1172/JCI26498)

Kang HM, Kang Y, Chun HJ, Jeong JW \& Park C 2013 Evaluation of the in vitro and in vivo angiogenic effects of exendin-4. Biochemical and Biophysical Research Communications 434 150-154. (https://doi. org/10.1016/j.bbrc.2013.03.053)

Karpe F, Fielding BA, Ilic V, Macdonald IA, Summers LKM \& Frayn KN 2002 Impaired postprandial adipose tissue blood flow response is related to aspects of insulin sensitivity. Diabetes $\mathbf{5 1} 2467-2473$. (https://doi.org/10.2337/diabetes.51.8.2467)

Kihira Y, Miyake M, Hirata M, Hoshina Y, Kato K, Shirakawa H, Sakaue H, Yamano N, Izawa-Ishizawa Y, Ishizawa K, et al. 2014 Deletion of hypoxia-inducible factor-1alpha in adipocytes enhances glucagon-like 
peptide- 1 secretion and reduces adipose tissue inflammation. PLoS ONE 9 e93856. (https://doi.org/10.1371/journal.pone.0093856)

Kinugasa M, Amano H, Satomi-Kobayashi S, Nakayama K, Miyata M, Kubo Y, Nagamatsu Y, Kurogane Y, Kureha F, Yamana S, et al. 2012 Necl-5/poliovirus receptor interacts with VEGFR2 and regulates VEGFinduced angiogenesis. Circulation Research 110 716-726. (https://doi. org/10.1161/CIRCRESAHA.111.256834)

Lee YS \& Jun HS 2016 Anti-inflammatory effects of GLP-1-based therapies beyond glucose control. Mediators of Inflammation 2016 1-11. (https://doi.org/10.1155/2016/3094642)

Lee Y-S, Park M-S, Choung J-S, Kim S-S, Oh H-H, Choi C-S, Ha S-Y, Kang Y, Kim Y \& Jun H-S 2012 Glucagon-like peptide-1 inhibits adipose tissue macrophage infiltration and inflammation in an obese mouse model of diabetes. Diabetologia 55 2456-2468. (https://doi. org/10.1007/s00125-012-2592-3)

Lei Y, Yang G, Hu L, Piao L, Inoue A, Jiang H, Sasaki T, Zhao G, Yisireyili M, Yu C, et al. 2017 Increased dipeptidyl peptidase-4 accelerates diet-related vascular aging and atherosclerosis in ApoEdeficient mice under chronic stress. International Journal of Cardiology 243 413-420. (https://doi.org/10.1016/j.ijcard.2017.05.062)

Liu FQ, Zhang XL, Gong L, Wang XP, Wang J, Hou XG, Sun Y, Qin WD, Wei SJ, Zhang Y, et al. 2011 Glucagon-like peptide 1 protects microvascular endothelial cells by inactivating the PARP-1/iNOS/NO pathway. Molecular and Cellular Endocrinology 339 25-33. (https://doi. org/10.1016/j.mce.2011.03.007)

Nauck MA, Duran S, Kim D, Johns D, Northrup J, Festa A, Brodows R \& Trautmann M 2007 A comparison of twice-daily exenatide and biphasic insulin aspart in patients with type 2 diabetes who were suboptimally controlled with sulfonylurea and metformin: a noninferiority study. Diabetologia 50 259-267. (https://doi.org/10.1007/ s00125-006-0510-2)

Nishimura S, Manabe I, Nagasaki M, Hosoya Y, Yamashita H, Fujita H, Ohsugi M, Tobe K, Kadowaki T, Nagai R, et al. 2007 Adipogenesis in obesity requires close interplay between differentiating adipocytes, stromal cells, and blood vessels. Diabetes 56 1517-1526. (https://doi. org/10.2337/db06-1749)

Olefsky JM \& Glass CK 2010 Macrophages, inflammation, and insulin resistance. Annual Review of Physiology 72 219-246. (https://doi. org/10.1146/annurev-physiol-021909-135846)

Pang C, Gao Z, Yin J, Zhang J, Jia W \& Ye J 2008 Macrophage infiltration into adipose tissue may promote angiogenesis for adipose tissue remodeling in obesity. American Journal of Physiology: Endocrinology and Metabolism 295 E313-E322. (https://doi.org/10.1152/ ajpendo.90296.2008)

Pasarica M, Sereda OR, Redman LM, Albarado DC, Hymel DT, Roan LE, Rood JC, Burk DH \& Smith SR 2009 Reduced adipose tissue oxygenation in human obesity: evidence for rarefaction, macrophage chemotaxis, and inflammation without an angiogenic response. Diabetes 58 718-725. (https://doi.org/10.2337/db08-1098)

Piao L, Zhao G, Zhu E, Inoue A, Shibata R, Lei Y, Hu L, Yu C, Yang G, Wu $\mathrm{H}$, et al. 2017 Chronic psychological stress accelerates vascular senescence and impairs ischemia-induced neovascularization: the role of dipeptidyl peptidase-4/glucagon-like peptide-1-adiponectin axis. Journal of the American Heart Association 6 e006421. (https://doi. org/10.1161/JAHA.117.006421)

Rausch ME, Weisberg S, Vardhana P \& Tortoriello DV 2008 Obesity in C57BL/6 $\mathrm{J}$ mice is characterized by adipose tissue hypoxia and cytotoxic T-cell infiltration. International Journal of Obesity $\mathbf{3 2}$ 451-463. (https://doi.org/10.1038/sj.ijo.0803744)
Shyangdan DS, Royle PL, Clar C, Sharma P \& Waugh NR 2010 Glucagonlike peptide analogues for type 2 diabetes mellitus: systematic review and meta-analysis. BMC Endocrine Disorders 10 20. (https://doi. org/10.1186/1472-6823-10-20)

Spencer M, Unal R, Zhu B, Rasouli N, McGehee RE, Peterson CA \& Kern PA 2011 Adipose tissue extracellular matrix and vascular abnormalities in obesity and insulin resistance. Journal of Clinical Endocrinology and Metabolism 96 E1990-E1998. (https://doi. org/10.1210/jc.2011-1567)

Takikawa A, Mahmood A, Nawaz A, Kado T, Okabe K, Yamamoto S, Aminuddin A, Senda S, Tsuneyama K, Ikutani M, et al. 2016 HIF1alpha in myeloid cells promotes adipose tissue remodeling toward insulin resistance. Diabetes 65 3649-3659. (https://doi.org/10.2337/ db16-0012)

Trayhurn P \& Wood IS 2004 Adipokines: inflammation and the pleiotropic role of white adipose tissue. British Journal of Nutrition 92 347-355. (https://doi.org/10.1079/BJN20041213)

Wang B, Wood IS \& Trayhurn P 2007 Dysregulation of the expression and secretion of inflammation-related adipokines by hypoxia in human adipocytes. Pflügers Archiv: European Journal of Physiology 455 479-492. (https://doi.org/10.1007/s00424-007-0301-8)

Weisberg SP, McCann D, Desai M, Rosenbaum M, Leibel RL \& Ferrante AW 2003 Obesity is associated with macrophage accumulation in adipose tissue. Journal of Clinical Investigation $\mathbf{1 1 2}$ 1796-1808. (https://doi.org/10.1172/JCI19246)

Wu JD, Xu XH, Zhu J, Ding B, Du TX, Gao G, Mao XM, Ye L, Lee KO \& Ma JH 2011 Effect of exenatide on inflammatory and oxidative stress markers in patients with type 2 diabetes mellitus. Diabetes Technology and Therapeutics 13 143-148. (https://doi.org/10.1089/dia.2010.0048)

Xie XT, Mo Z, Chen K, He H \& Xie Y 2011 Salvage of a femoral nonunion after primary non-Hodgkin's lymphoma of bone: a case report and literature review. Medical Science Monitor 17 CS138-CS143. (https:// doi.org/10.12659/MSM.882035)

Xu F, Burk D, Gao Z, Yin J, Zhang X, Weng J \& Ye J 2012 Angiogenic deficiency and adipose tissue dysfunction are associated with macrophage malfunction in SIRT1-/- mice. Endocrinology 153 1706-1716. (https://doi.org/10.1210/en.2011-1667)

Xu F, Lin B, Zheng X, Chen Z, Cao H, Xu H, Liang H \& Weng J 2016 GLP-1 receptor agonist promotes brown remodelling in mouse white adipose tissue through SIRT1. Diabetologia 59 1059-1069. (https://doi. org/10.1007/s00125-016-3896-5)

Yang G, Lei Y, Inoue A, Piao L, Hu L, Jiang H, Sasaki T, Wu H, Xu W, Yu C, et al. 2017 Exenatide mitigated diet-induced vascular aging and atherosclerotic plaque growth in ApoE-deficient mice under chronic stress. Atherosclerosis 264 1-10. (https://doi.org/10.1016/j. atherosclerosis.2017.07.014)

Ye J 2011 Adipose tissue vascularization: its role in chronic inflammation. Current Diabetes Reports 11 203-210. (https://doi.org/10.1007/s11892011-0183-1)

Ye J, Gao Z, Yin J \& He Q 2007 Hypoxia is a potential risk factor for chronic inflammation and adiponectin reduction in adipose tissue of ob/ob and dietary obese mice. American Journal of Physiology: Endocrinology and Metabolism 293 E1118-E1128. (https://doi. org/10.1152/ajpendo.00435.2007)

Zinman B, Hoogwerf BJ, Durán García S, Milton DR, Giaconia JM, Kim DD, Trautmann ME \& Brodows RG 2007 The effect of adding exenatide to a thiazolidinedione in suboptimally controlled type 2 diabetes: a randomized trial. Annals of Internal Medicine 146 477-485. (https://doi.org/10.7326/0003-4819-146-7-200704030-00003)

Received in final form 13 May 2019

Accepted 28 May 2019

Accepted Preprint published online 28 May 2019 https://joe.bioscientifica.com https://doi.org/10.1530/JOE-18-0639 (c) 2019 Society for Endocrinology Published by Bioscientifica Ltd. Printed in Great Britain 